\title{
Using Light Polarization in Laser Scanning
}

\author{
J. Clark, E. Trucco and H-F. Cheung \\ Department of Computing and Electrical Engineering \\ Heriot-Watt University, Edinburgh \\ Scotland, EH14 4AS
}

\begin{abstract}
We use polarization analysis in triangulation-based laser scanners to disambiguate the true laser stripe from spurious inter-reflections caused by holes and concavities on metal surfaces. Stripe candidates are discriminated by projecting linearly polarised laser light and measuring the polarization state of the linearly polarized component of the observed stripe candidates. Initial experimental results are reported and discussed.
\end{abstract}

\section{Introduction}

Laser-based triangulation range sensors $[4,5,6,10]$ project a sheet of laser light onto a scene, observe the resulting 3 -D curve (stripe) through calibrated cameras, and compute the 3-D position of the stripe points by triangulation. The cameras are arranged so that the image of the stripe intersects each image row (or column) at most once, and the range $Z$ can be linked directly to one image coordinate.

The detection of stripe points in the image is critical. A popular method is to scan the image rows (or columns) looking for the peak of the intensity signal, then locate its position with subpixel accuracy $[3,4,5]$. However, when the surfaces observed are highly specularly reflective, the laser stripe can be reflected onto different parts of the surface in view, creating several peaks. If the wrong peak is chosen, the triangulation yields wrong range measures. This problem occurs typically with manufactured metal components, on which we have concentrated. Figure (1(d) shows an example of wrong range data caused by inter-reflections within the concavity of the metal part modelled in Figure1(a). Figure 1c shows an image with multiple stripe reflections. Shiny components are often sprayed with matte paint to suppress inter-reflections (Figure 1(b)), but this is obviously not always possible or desirable. The range images were acquired in our laboratory [10].

Ideally, scanners should distinguish automatically the primary reflection of the laser stripe from its subsequent reflections. Trucco and Fisher [5] obtained good results by exploiting geometric constraints, but their method simply rejects all inconsistent measurements without identifying the true stripe. We propose a solution based on polarization. The parameters of incident linearly polarized light (primarily its orientation, or phase) change upon each specular reflection on metal surfaces [8]. This can help to disambiguate the true laser stripe from 


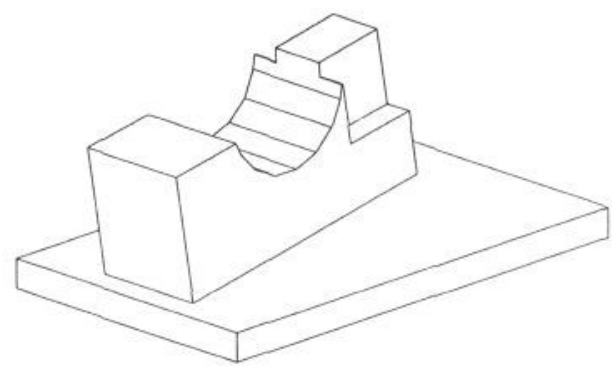

(a)

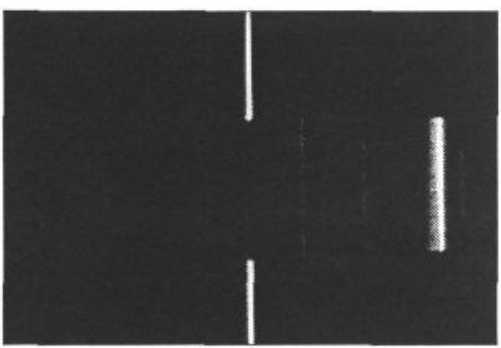

(c)

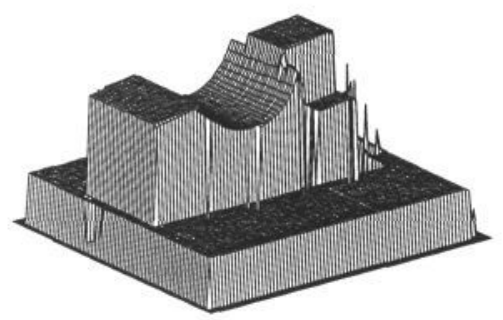

(b)

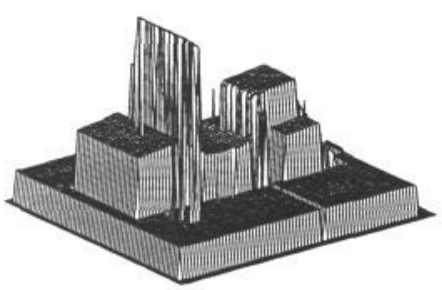

(d)

Figure 1: (a) CAD model of metal part; (b) range image of matte-sprayed part; (c) multiple stripes observed in concavity of shiny part; (d) range image of shiny part

subsequent inter-reflections caused by holes and concavities of the metal surfaces. This takes polarization vision into range sensing, adding, to our best knowledge, a new application to those already explored (e.g. shape analysis, material separation, and occluding contour detection $[7,8,9])$, and providing a promising technique for improving the reliability of laser scanners when used to measure metal components.

\section{Characterising polarization states}

A ray of light can be described by its electric field waveform $\mathbf{E}(t)$ and magnetic field waveform $\mathbf{H}(t)$, oscillating at the same frequency but orthogonally to each other [1]. At each instant $t$, the plane defined by $\mathbf{E}(t)$ and $\mathbf{H}(t)$ is perpendicular to the propagation direction of the ray itself, therefore $\mathbf{E}(t)$ alone is a sufficient representation of the light. Different polarization states can be described by decomposing $\mathbf{E}(t)$ in two mutually orthogonal components $E_{x}(t)$ and $E_{y}(t)$ in the plane defined by $\mathbf{E}$ and $\mathbf{H}$. Unpolarized light results from the non-deterministic superposition of $E_{x}(t)$ and $E_{y}(t)$, whereby $\mathbf{E}(t)$ can be described only statistically. When its two components oscillate in phase, light becomes linearly polarized, and 
the tip of $\mathbf{E}(t)$ oscillates on a fixed line. Any other phase difference produces $\mathrm{el}$ liptically polarized light, i.e. the tip of $\mathbf{E}(t)$ follows an ellipse in the $\mathbf{E} \mathbf{H}$ plane. In general, light is often partially polarized, and can be regarded as the sum (superposition) of completely polarized and unpolarized light. In this paper, we refer to the orientation of partially polarized light meaning the orientation of its linearly polarized component.

Linearly polarized light can be obtained by passing unpolarized light through a linear polarizer, a filter characterised by the orientation $\psi$ of the plane in which the electric field is forced to oscillate, measured with respect to a reference direction in the $\mathbf{E ~} \mathbf{H}$ plane. If linearly partially polarized light of orientation $\theta$ is passed through a linear polarizer of orientation $\psi$, the intensity of the resulting polarized light is a function of $(\theta-\psi)$ [1] called the transmitted radiance sinusoid (TRS) and given by

$$
\frac{I_{\max }+I_{\min }}{2}+\frac{I_{\max }-I_{\min }}{2} \cos (2 \psi-2 \theta)
$$

Experimental TRS estimation is done by acquiring three component images through a linear polarizer at three orientations $\psi=0^{\circ}, 45^{\circ}$ and $90^{\circ}$. Since three points identify a sinusoid completely, the three intensity values $I_{0}(i, j), I_{45}(i, j)$ and $I_{90}(i, j)$ measured at each pixel $(i, j)$ suffice to reconstruct the TRS at that pixel [9]. The TRS parameters can then be used to characterise partial polarization states through partial polarization $\frac{I_{\max }-I_{\min }}{I_{\max }+I_{\min }}$ ( 0 for unpolarized light, 1 for linearly polarized light), total radiance $I_{\max }+I_{\min }$, and phase $\theta$ (taken with respect to a fixed reference in the $\mathbf{E} \mathbf{H}$ plane). Wolff and Mancini [9] give closed-form formulae to obtain phase, total radiance and partial polarization from $I_{0}(i, j), I_{45}(i, j)$ and $I_{90}(i, j)$. Comparison of the fitting accuracy of Wolff's formulae with nonlinear optimisation methods revealed no significant differences.

Figure 2 shows the experimental setup used throughout our experiments, in practice a simple laser triangulation sensor "augmented" with polarization analysis equipment. Laser light generated by a diode laser $(\lambda=670 \mathrm{~nm})$ is collimated, expanded into a stripe by a cylindrical rod lens and then projected onto the object being observed. A linear polarizer is placed between the collimating optics and the rod lens to allow control of the plane of polarization.

A second linear polarizer is placed in front of the camera and is rotated at three different angles to acquire the component images. A neutral density filter is used to reduce the risk of CCD saturation caused by intense specular reflections of the laser. As the spectral range of the CCD camera extends to $1100 \mathrm{~nm}$ and the efficiency of the polarizer is reduced at wavelengths beyond $750 \mathrm{~nm}$, an infra-red filter is fitted to the camera to prevent transmission of unwanted wavelengths. A Pulnix TM500 camera (fitted with a $25 \mathrm{~mm}$ focal length lens) and Data Translation DT2867 framestore are used to acquire $512 \times 768,8$-bit images.

\section{Discriminating inter-reflections}

Spurious inter-reflections of the laser light can be separated from the true stripe (primary reflection) as the orientation of the linearly polarized component of the incident light changes upon specular reflection on metal surface (aluminium in 


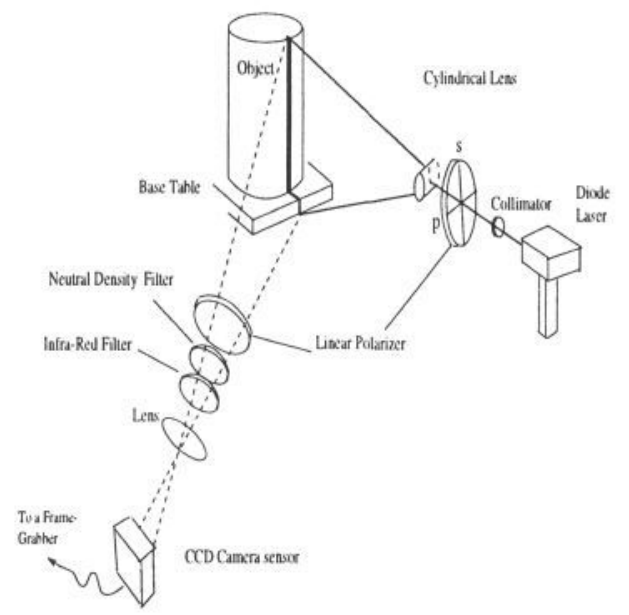

Figure 2: Experimental polarization analysis and triangulation geometry.

our experiments). The change can be measured by estimating a TRS at each stripe pixel. Throughout our experiments, we measured orientation differences between true and spurious stripes large enough to allow easy separation. However, the physical phenomena underlying the method are not trivial. Our explanation is based on the Torrance-Sparrow [2] and Fresnel reflectance models [8]. Full experimental verification is still under way at the moment of writing.

According to the Torrance-Sparrow model, incident laser light is reflected specularly by planar microfacets modelling the surface roughness [2]. The normals of visible microfacets always lie in the plane defined by the camera's viewing direction and the ray of laser light (Figure 3 ). Therefore, the specular plane of incidence at any stripe point is defined by the laser direction and the camera's viewing direction, independent of the surface normal. This means that, at least for single reflections, we can arrange the plane of laser light and the camera viewing direction at an optimal angle, such that significant orientation changes are guaranteed, independent of surface normals. Of course the latter can still be estimated after collecting range measurements by triangulation.

Since the laser stripe spans a certain visible length $L$ (depending on the camera's focal length and the stand-off distance $H$ ), the specular plane will actually change across the stripe ( $\alpha$ in Figure 3), pivoting around the line through the lens centre and the laser source. However, in our setup $H \cong 1000 \mathrm{~mm}$ and $L \leq 200 \mathrm{~mm}$, leading to a worst variation $\alpha \leq 10^{\circ}$; this means that the orientation of the linearly polarized light can be regarded as fixed with respect to all "effective" specular planes within good approximation.

Specular reflection of linearly polarized light on metal introduces some phase retardation between $E_{x}(t)$ and $E_{y}(t)$, so that the polarized component of the reflected light is actually elliptical and a decrease in partial polarization occurs. Consequently, the projection of the electric field along the polarizer's direction (on which the measured intensity depends) does not vary as $\cos \theta$ (linearly polarized light), $\theta$ being the angle formed by the electric field and the polarizer's direction, 


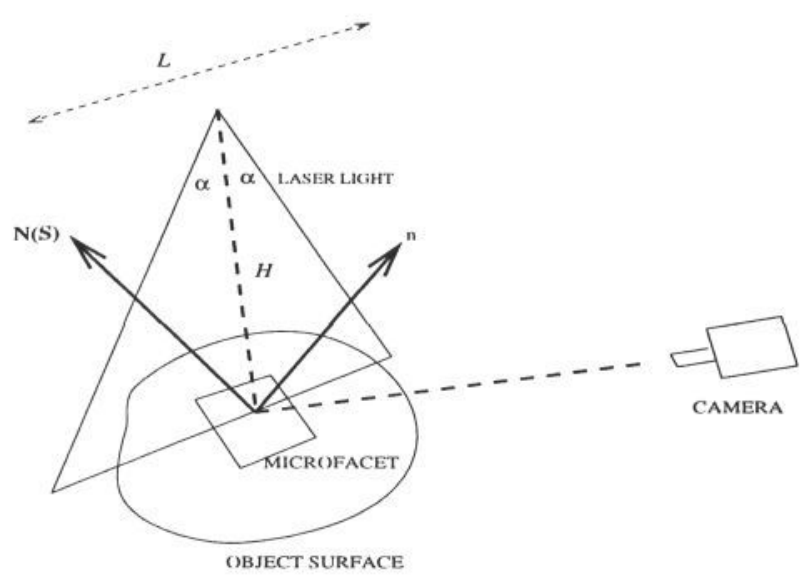

Figure 3: Difference between surface normal $\mathbf{N}_{S}$ and microfacet normal $\mathbf{n}$.

but as $2 \sqrt{a \cos ^{2} \theta+b \sin ^{2} \theta}$, where $a$ and $b$ are the ellipse semiaxes and $a>b$, and the intensity profile is not the expected TRS. Figure 4 shows the length of the projection of the ellipse onto the linear polarizer direction as a function of $\theta$ and resulting intensity (dotted), and the length of the projection of a linearly polarized electric field and resulting intensity (solid) for varying ellipticity $\frac{b}{a}$. If the ellipticity is small ( $b<<a$, i.e. small phase retardation), the polarized component can be considered linear with good approximation for most $\theta$, and the TRS is still a reasonable characterisation of the polarized component.

The Fresnel reflectance model predicts that the amount of retardation, and therefore the ellipticity [1] of the polarized component, varies with angle of incidence and relative orientation of the incident linearly polarized light and the plane of incidence. The predicted ellipticity for single specular reflections on aluminium as function of angle of incidence and orientation of incident linearly polarized light relative to p-polarization direction is shown in Figure 5(a): ellipticity remains small for a considerable range of angles of incidence, which can be controlled if referred to single reflections off microfacets as described above.

In summary, we expect that the polarized component of the reflected light is practically linear and its orientation predictable by the Fresnel reflectance model, assuming that (a) the polarization state of the observed light is due predominantly to single specular reflections off microfacets, (b) the angle of incidence with visible microfacets is roughly constant, (c) orientation of the linearly polarized incident laser light is known. Figure 5(b) shows the predicted rotation of the light after single specular reflection on aluminium, which makes it possible to identify the true stripe in the image. In practice the predicted values provide a rough indication to identify the primary reflection.

Further experimentation is needed to assess the exact conditions of validity of our model. For instance, geometric reflectance models work as long as the average size of the microfacets is larger than the light's wavelength, in our case $670 \mathrm{~nm}$; behaviour with varying roughness (to be measured with a stylus profilometer) must be tested. The effect of surface roughness is often approximated by considering 

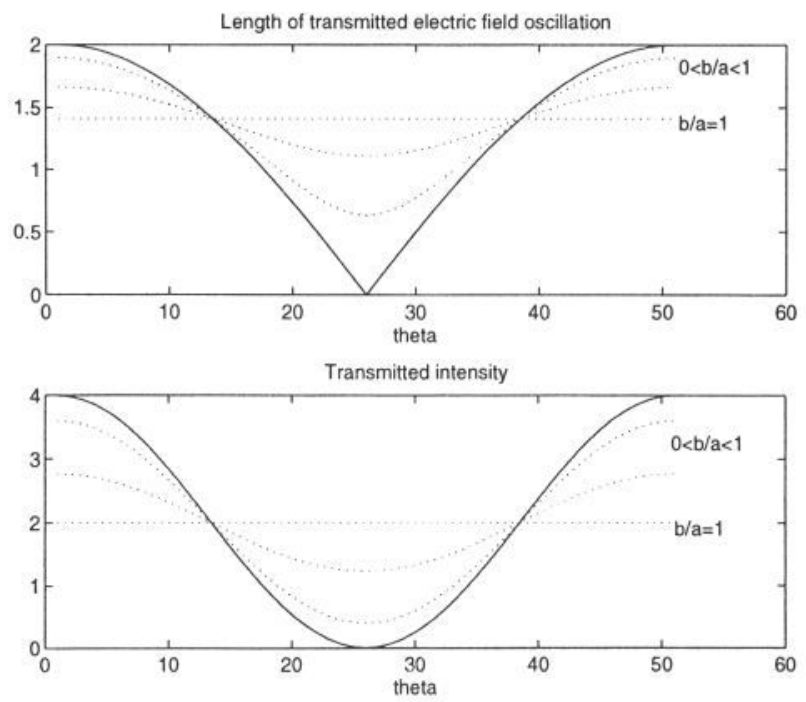

Figure 4: Length of projection of linearly polarized E (solid) along polarizer's direction, and resulting intensity (below) vs. length of projection of elliptically polarized $\mathbf{E}$ (dotted) and resulting intensity. Ellipticity varies btw 0 (linearly polarized) and 1 (circularly polarized).

a thin film coating the surface; Figure 5 includes one example of predictions for such a case. A part of the observed light is contributed by multiple specular interreflections with two or more microfacets; we expect this light to be practically unpolarized [8]. Other factors still unquantified and limiting the accuracy of the method include imperfect collimation of the laser light, and the effect of the milling texture of some metallic components.

\section{Experimental results}

We experimented with shiny aluminium objects with holes and concavities, to create strong specular inter-reflections of the primary stripe. The resulting intensity profiles depend on the position and geometry of stripe and surfaces, making it usually impossible to tell stripes apart from the stripe intensity only.

In a first series of experiments, we created secondary reflections of various widths and intensities by projecting laser light onto an aluminium plate, and observing the specular inter-reflections on a second aluminium plate forming various angles with the first one. An example is shown in Figure 6(a). The secundary reflection (right) of the stripe is very strong and widespread. Figure 6(c) plots the estimated polarization parameters on a horizontal scanline across the image (since all the components appear in the same graph, the units of the $y$ axis are meaningless). Notice the two "ghost" intensity peaks of the inter-reflection (dotted line). An intensity-based peak detector would register three peaks of similar intensity, all plausible candidates for the true stripe. Using polarization, the phase 


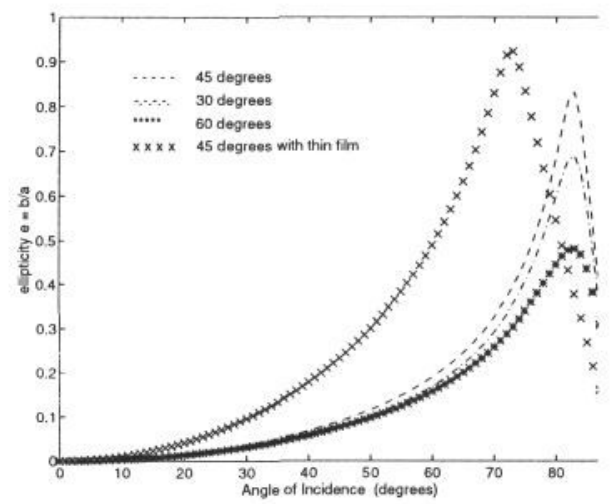

(a):ellipticity.

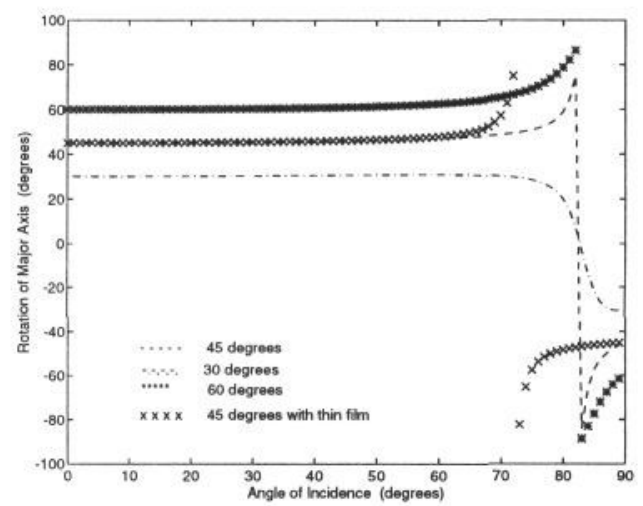

(b): rotation.

Figure 5: Ellipticity (a) and predicted rotation (b) of elliptically polarized light after single specular reflection on aluminium, as function of angle of incidence and orientation of incident light relative to p-polarization direction.

shift between primary and secondary inter-reflections (solid line) is very obvious. As in most laser triangulation systems, we assume that the stripe appears brighter than the background and can be roughly located by intensity thresholding. The true stripe located by the system is shown in Figure 6(b).

In another series of experiments we found that the true stripe could be discriminated from higher-order inter-reflections such as those produced by holes, although the light behaviour is far less predictable in this case [8]. Laser light can bounce off the hole's bottom and walls several times, become visible on the hole's wall with unpredictable intensity, and confuse intensity-based stripe location. Figure 7 (a) shows an example of intensity image with a conical hole. Figure $7(\mathrm{c})$ shows the polarization parameters along a horizontal scanline of the image. Figure 7(b) shows the result of polarization-based disambiguation.

To test our method with controlled higher-order inter-reflections, we projected the polarized stripe onto a high quality elliptical mirror, arranged so that three subsequent reflections would appear (Figure 8). In the image shown in Figure 9(a), the true stripe crosses the image vertically on the right. The stripe is reflected first off the horizontal plane (barely visible at the bottom), then onto the curved surface (middle left). The segmentation is shown in Figure $9(\mathrm{~b})$.

\section{Discussion}

We have presented an initial study on polarization-based vision for triangulationbased laser scanners. The purpose was to separate the true laser stripe from its spurious inter-reflections on metal surfaces. The technique could have wide applicative interest, as both laser range scanners and highly reflective metal objects occur frequently in applications. Our results identify the method as a very interesting candidate for increasing the robustness of laser triangulation sensors. Polarization-based stripe detection is based on the physical properties of reflected 
light, therefore more general and less dependent on the geometry of the observed surfaces than intensity-based methods like [5].

Future work includes refining and validating experimentally the theoretical model of the underlying physical phenomena; installing a liquid crystal polarization optical head [9] to improve the accuracy of TRS estimates; investigating the performance of the method with different metals; and integrating laser triangulation and polarization analysis on one of the range scanners available in our lab [10].

\section{Acknowledgements}

Larry Wolff provided invaluable encouragement, practical help, and comments. Jim Clark was supported by UK EPSRC Grant GR/J07891.

\section{References}

[1] R. M. A. Azzam and N. M. Bashara, Ellipsometry and Polarized Light, North Holland, Amsterdam, 1977.

[2] R. Cook and K. Torrance: A Reflectance Model for Computer Graphics, Journal of Computer Graphics, vol. 15, 1981, pp. 245 - 263.

[3] D. K. Naidu and R. B. Fisher, A Comparative Analysis of Algorithms for Determining the Peak Position of a Stripe to Subpixel Accuracy, Proc. British Machine Vision Conf., 1991, pp. 217 - 225.

[4] P. Saint-Marc, J.-L. Jezouin and G. Medioni, A Versatile PC-Based Range Finding System, IEEE Trans. Robot. Autom., vol. RA-7, no. 2, April 1991, pp. $250-256$.

[5] E. Trucco and R. B. Fisher, Acquisition of Consistent Range Data Using Local Calibration, Proc. IEEE Int. Conf. Rob. Autom., San Diego, 1994, pp. 3410 - 3415 .

[6] E. Trucco, R. B. Fisher and A.W. Fitzgibbon: Direct Calibration and Data Consistency in 3-D Laser Scanning, Proc. British Machine Vision Conference BMVC94, York, September 1994, pp. 489 - 498.

[7] L. B. Wolff: Polarization-Based Material Classification from Specular Reflection, IEEE Transactions on Patt. Anal. Mach. Intell., vol. 12(11), 1990, pp. 1059 - 1071.

[8] L. B. Wolff: Polarization Methods in Computer Vision, PhD Thesis, Columbia University, 1991.

[9] L. B. Wolff and T. A. Mancini: Liquid Crystal Polarization Camera, Proc. IEEE Workshop on Applications of Computer Vision, 1992, pp. 120 - 127.

[10] J. Clark, G.H. Zhang, A. M. Wallace, Image Acquisition Using Fixed and Variable Triangulation, Proc. IEE Int. Conf. on Image Processing and Applications, Edinburgh, July 1995, pp. $539-543$. 


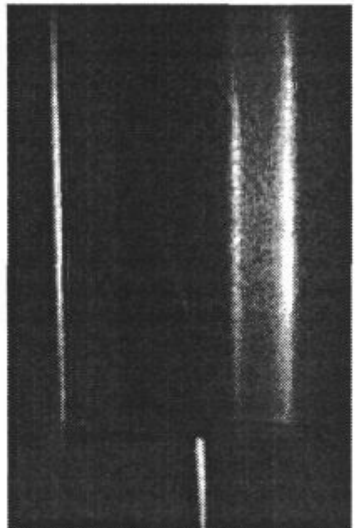

(a):Intensity image. (b): Segmented image.

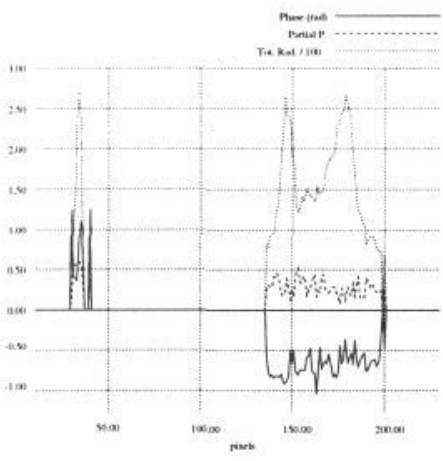

(c): Polar. param.s along scanline.

Figure 6: Recovery of true stripe from reflections created with two metal plates.

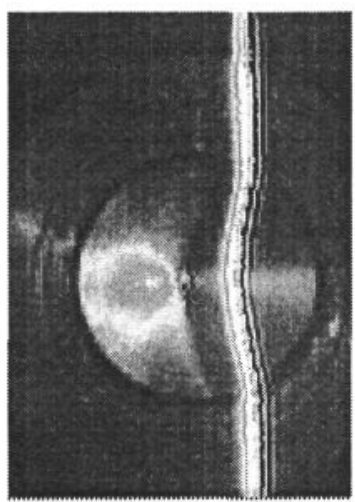

(a):Intensity image.

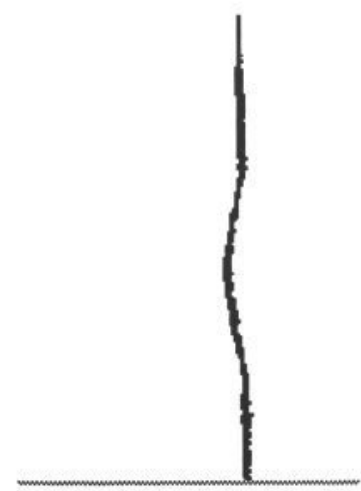

(b): Segmented image.

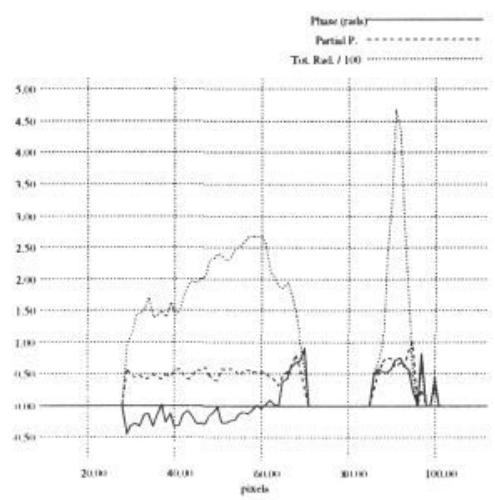

(c): Polarization params along scanline.

Figure 7: Recovery of true stripe from reflections created by a conical hole in a metal surface.

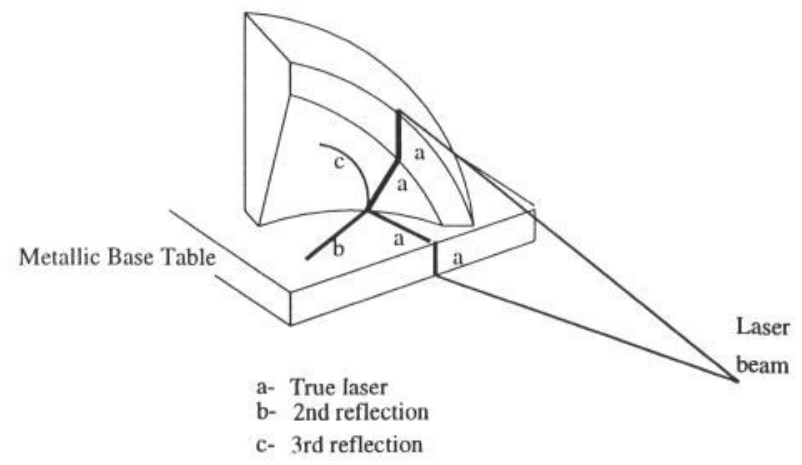

Figure 8: Experimental arrangement using a mirror to create multiple reflections. 


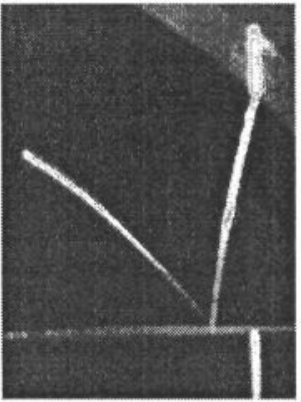

(a):Intensity image.
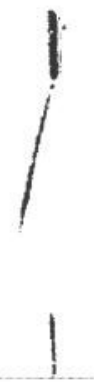

(b): Segmented image.

Figure 9: Recovery of true stripe with inter-reflections created by an elliptical mirror. 